\title{
Evidence for Lectin Signaling to the Nuclear Matrix: Cellular Interpretation of the Glycocode
}

\author{
Christopher H. Chay* and Kenneth J. Pienta \\ Department of Internal Medicine, University of Michigan Comprehensive Cancer Center and Division of \\ Hematology/Oncology, Ann Arbor, Michigan 48109-0946
}

All cells exist within a context. They are often in contact with other cells or they may be bound by the extracellular matrix (ECM). The microenvironment around a cell, including interactions with neighboring cells and the ECM, has been shown to effect morphology, DNA synthesis, and even gene expression [Gospodarowicz et al., 1978; Bissell et al., 1981]. These signals dictate how a cell responds to its surroundings. In normal tissue, cells are governed by a given set of rules such as contact inhibition and apoptosis, but transformed cells disregard these rules by forming clumps of cells, moving through complex matrices, and ignoring commands for programmed cell death. Understanding what messages cells obtain from their surroundings, how they interpret these messages, and how they respond is an important dynamic of both normal and pathologic cell biology.

The tissue matrix is the physical framework connecting the components of a cell with the ECM [Isaacs et al., 1981; Fey et al., 1984; Pienta et al., 1993]. The tissue matrix is a three dimensional structure which links the chromosomes to the nuclear matrix, followed by the cytoskeleton, membrane matrix, and ECM. The nuclear matrix has several functions including determining nuclear morphology [Berezney and Coffey, 1974], providing DNA organization and scaffolding [Pienta et al., 1991], replicating DNA [Vogelstein et al., 1980], and transcribing RNA as well as directing RNA transport [Ciejek et al., 1982]. The cytoskeletal matrix is composed of intermediate filaments (IFs), actin

\footnotetext{
*Correspondence to: Christopher H. Chay, CCGC Building, Room 7303, University of Michigan, 1500 East Medical Center Drive, Ann Arbor, MI 48109-0946.

E-mail: cchay@umich.edu

Received 17 October 2000; Accepted 18 October 2000

(c) 2001 Wiley-Liss, Inc.
}

microfilaments, and microtubules and plays a role in cytokinesis and mitosis. IFs specifically have been shown to provide direct contact between the nuclear matrix and the cytoskeleton.

A cell interacts with its microenvironment through the membrane matrix, proteins and glycoproteins imbedded in the plasma membrane. Protein-protein interactions have been shown to be an important component not only for cellular adhesion, but also for signal transduction. Cell surface integrins, a family of heterodimeric glycoproteins, have been well described as binding to specific RGD sequences found in collagen and other membrane proteins [Ruoslahti and Obrink, 1996; Gahmberg et al., 1997]. The cytoplasmic domains of integrins have been shown to bind to essential cytoskeletal proteins such as talin and $\alpha$-actinin, and activate focal adhesion kinase in creating adhesion complexes. By interacting with the ECM and neighboring cells through the adhesive properties of its external domain, and the cytoskeletal framework with its cytoplasmic domain, integrins demonstrate an ability to bridge the gap between mechanical adhesion and intracellular signaling.

While much focus in the field of cell adhesion and ECM interactions has been placed on integrins and protein-protein interactions, we are only just beginning to understand the spectrum of protein-carbohydrate interactions. Breaking the DNA and polypeptide codes has allowed the in-depth study of the passage of genetic material, mutations of this system, and its expression into proteins. Both DNA and polypeptides represent linear codes with permutations of a limited set of units. Carbohydrates, by containing branched chains and additional sulfate, phosphate, or $O$-acetyl groups, have a variety of organizational 
combinations that allow for an impressive array of three dimensional structures [Sharon and Lis, 1997]. For only three hexose monosaccharides, there are 1,056 potential oligosaccharides, and with the addition of each sugar unit there is an exponential increase in the number of possible structures. The potential biologic diversity of these carbohydrates, in terms of sequence and conformation, makes it an attractive system for conveying biologic information, and was initially called the glycocode by Kasai and Hirabayashi [1996]. With the ubiquitous presence of carbohydrates on cell surface glycoproteins as well as throughout the ECM, it is likely that cells use this glycocode to interpret their surroundings, bind with increased specificity, and interact with surrounding cells.

\section{LECTINS}

Lectins represent a diverse category of carbohydrate binding proteins [Gabius, 1994, 1997]. Lectins have highly conserved carbohydrate binding regions and have been identified in a broad range of multicellular organisms ranging from nematodes to humans [Kasai and Hirabayashi, 1996]. Five distinct types of lectins have been described including the C-type, the Ptype, the I-type, pentraxins, and galectins (Stype). They share the property of having con- served carbohydrate recognition domains (CRDs), but with different binding motifs and target ligands they clearly represent distinct families [Drickamer, 1995].

Lectins have many ways of binding to their ligand (Fig. 1) [Kaltner and Stierstorfer, 1998]. Simple models may include interactions between a cell-bound lectin to a carbohydrate found on another cell or the ECM. To add a level of complexity, soluble carbohydrate moiety may bind lectins on two surfaces, or even on the same surface, which is known as "bridging." In addition, some lectins have been shown to bind both carbohydrates and proteins in a non-competitive fashion. Lectins are also secreted where they may interact in a paracrine or autocrine fashion.

The C-type lectins are distinguished in their requirement for $\mathrm{Ca}^{2+}$ ions for sugar binding, in addition to having a highly conserved CRD domain [Drickamer, 1993]. This family of lectins includes the selectins ( $\mathrm{L}, \mathrm{P}$, and $\mathrm{E}$ ), as well as CD 69, CD 72, and the Asialoglycoprotein receptor. P-type lectins have been shown to be involved in routing lysosomal enzymes and are thought to orient enzymes that can degrade proteoglycans in the ECM [Kornfeld, 1992]. The I-type lectins share a characteristic immunoglobulin-like CRD. I-type lectins can undergo homophilic and heterophilic interactions

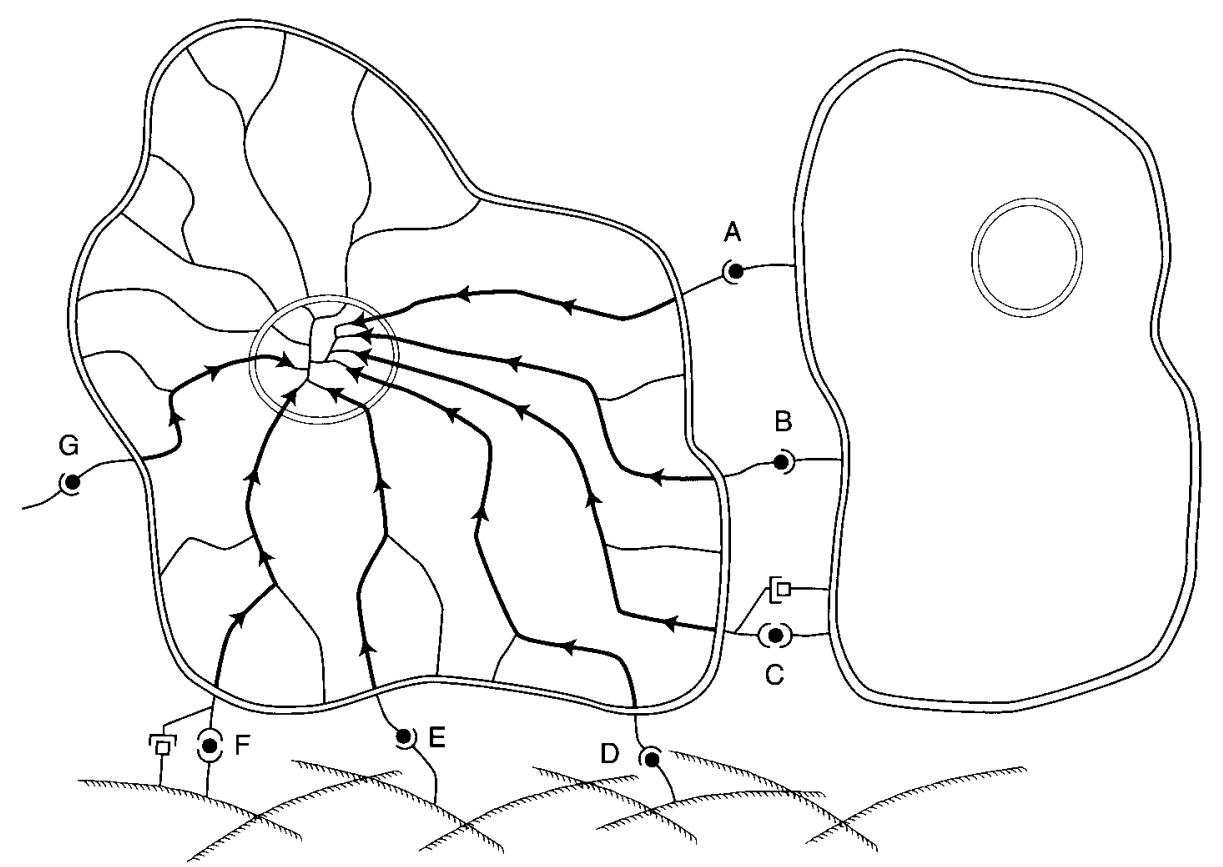

Fig. 1. Lectin signaling to the nuclear matrix through possible pathways. Cell-cell interactions $(\mathrm{A}-\mathrm{C})$, cell-matrix interactions

(D-F), and solubilized (G). Lectins may also interact independently through carbohydrate and protein interactions ( $\mathrm{C}$ and F). 
[Zanetta, 1997] and include ICAM, PECAM, N$\mathrm{CAM}$, and $\mathrm{P}_{\mathrm{o}}$ glycoprotein. Pentraxins form a distinct pentameric configuration and include the acute phase reactants C-reactive protein (CRP) and serum amyloid P (SAP). Their ligands have been found on numerous microorganisms and CRP has been shown to activate the complement cascade.

Galectins share a conserved CRD and bind specifically to $\beta$-galactosides. Nine galectins have been identified to date, of them galectin-1 and -3 are the best studied. Galectin- 1 is ubiquitously found and because it exists as a homodimer it is thought to allow for carbohydrate cross-linking. Galectin-3 functions as a monomer, and has been shown to be present on the plasma membrane as well as secreted as a response to stress or inflammation [Sato and Hughes, 1994]. Galectin-3 has also been found on many tissues and it has been shown to be involved in a wide range of functions including cell adhesion [Sato and Hughes, 1992], angiogenesis [Nangia-Makker et al., 2000], and the recruitment of macrophages.

While galectins represent a relatively small family compared to the C-type lectins, their biologic functions tend to be less well defined. Even their cellular localization is controversial in that they have been identified both in the nucleus and the cytoplasm, as well as the plasma membrane and the ECM [Barondes, 1984; Cooper and Barondes, 1990]. As proposed by Kasai and Hirabayashi [1996], it is possible that lectins may represent a category of proteins which may transduce carbohydrate signals. We would propose that in relaying and interpreting the glycocode found as molecular signals in the microenvironment, lectins may alter the nuclear matrix through the tissue matrix to affect alterations in cellular response.

\section{Lectins and the Nuclear Matrix}

From what is known about lectins, it is clear that they are present on the plasma membrane or are secreted and they can interact with the ECM. Studies have shown that lectins are involved in a variety of functions, with galectins having the most diverse effects. The C-type and I-type lectins have been shown to be involved with different forms of cellular adhesion. The P-type lectins are involved in intracellular shuttling and ECM degradation. The role of the galectins remains somewhat less clear. They have been implicated in a wide variety of complex activities, which suggests that they act not only as sensors of the cellular microenvironment, but they may have the ability to interpret or at least transduce these signals.

The ECM has been shown to affect both the cytoskeleton and the nuclear matrix. Getzenberg et al. [1991] showed that the ECM can alter the tissue matrix as displayed on two-dimensional gels. This suggests that the cell receives signals as to its surrounding ECM and triggers specific changes in tissue matrix proteins. A recent study by Lelievre et al. [1998] has shown altered expression of specific nuclear matrix proteins with changes in the ECM. When a human mammary epithelial cell line (HMEC) was grown in three-dimensional reconstituted basement membrane, they noticed changes in cellular morphology. The nuclear matrix proteins NuMA, splicing factor SRm160, and Rb, showed altered spatial arrangement compared to nuclear lamina protein and lamin B, which remained unchanged. These studies support the finding that the ECM can affect the tissue matrix not only in terms of morphology, but spatial changes in specific nuclear matrix proteins have also been observed.

Changes in the nuclear matrix require some form of signaling from the ECM. There is evidence that lectins may transmit changes in the microenvironment either through classical signal transduction mechanisms or by altering the cytoskeleton. In the model of neutrophil adhesion, L-selectin, a C-type lectin, can activate the MAPK pathway through src-tyrosine kinase $\mathrm{p} 56^{\text {lck }}$ [Brenner et al., 1996; Villalobo and Gabius, 1998]. In addition, this stimulates Ras activation of Rac with signaling through JNK/ SAPK. These pathways lead to the activation of multiple transcription factors, which can result in proliferation, differentiation, and cellular activation.

In addition to classical signal transduction pathways there have been observations that lectins may alter the tissue matrix. Arena et al. [1990] treated cultured chick embryo skin fibroblasts with the lectins PNA and SBA. They observed that these exogenous lectins caused alterations in the arrangement of various cytoskeletal components including myosin, actin, and tubulin. This resulted in changes in the cellular morphology. More specifically, Fulgenzi et al. [1998] have observed that the lectin, comitin, can bind F-actin. Prior studies had shown an interaction between comitin and 
F-actin, but by electron microscopy they observed that comitin binds F-actin in areas distinct from actin binding proteins. This suggests that it may be important for the cell not to have comitin compete with other skeletal proteins due to its function. The third component of the cytoskeleton, IFs, is likely to play the most significant role in mediating lectin signaling. Traub et al. [1985] have suggested that IFs may even directly interact with chromosomes by showing that cytoplasmic adhesion molecules can bind DNA, RNA, and histones in vitro. This would suggest that IFs may bridge the gap between the cytoskeleton and signaling to components of the nuclear matrix to affect DNA transcriptional regulation, but specific sequences and regulatory processes have not yet been described.

Signaling from adhesion complexes involving both integrins and lectins in an integrated fashion has not only been shown to occur via cytoskeletal components, but studies suggest that components of these adhesion complexes may have a role directly in the nuclear matrix. Connective membrane skeletal (CMS) proteins help to form focal adhesion complexes, which combine integrins and lectins with cytoskeletal components and signal transducing kinases [Lelievre and Bissell, 1998]. These CMS proteins, including $\beta$-catenin, plakoglobin, plakophilin 2 , and zyxin, have been identified in the nucleus. In addition, some are simultaneously associated with the nucleus and the plasma membrane. The identification of CMS proteins in the nucleus has been observed in cells that do not use them in cellular adhesion, as well as in cells with disrupted adhesion complexes. This raises the question whether these proteins may act as pathways for the cytoskeleton to relay changes to the nucleus and possibly alter the nuclear matrix. The CMS protein $\beta$-catenin has been shown to form a complex with the transcription factor LEF-1 and bind to the $5^{\prime}$ end of the E-cadherin gene, suggesting one possible mechanism of cytoskeletal proteins affecting the nuclear matrix to modulate gene expression [Huber et al., 1996]. It is possible that these proteins may play other roles at various steps of gene expression such as transcriptional activation or suppression, nuclear trafficking of mRNA, and one could even envision actions on the spatial alignment of inducer sequences to promote gene expression and DNA organization.
While there is evidence of signaling by lectins via the tissue matrix, the more compelling effect on the nuclear matrix is the ability of lectins to alter cellular functions. Knowing that lectins may act in a paracrine fashion, [Inohara et al. [1998] have shown that soluble galectin-3 can stimulate cell proliferation. Not only was this response dose-dependent, but the effect could be blocked by competing away the lectin binding with lactose. In similar experiments they examined the effect of galectin-3 on the endothelial cell line HUV-EC-C [Nangia-Makker et al., 2000]. The addition of galectin-3 caused increased organization of the cells into tubules when cultured on matrigel. Again, this was dose-dependent and the effect could be competed away with lactose and an antibody to galectin-3. In addition, an in vivo matrigel assay was performed to assess neovascularization. They found no vessels in the matrigel injected under the skin of mice, whereas the matrigel imbedded with galectin-3 had an increased microvessel density suggesting a role for galectin-3 in angiogenesis.

Direct evidence of lectin involvement in the nuclear matrix has come from Wang et al. [1995]. In a series of experiments they demonstrated that galectin-3 is associated with the nuclear matrix. In addition, they showed that galectin-3 binds both ssDNA and RNA. Interestingly, these functions are not competed away with carbohydrates suggesting that a different region of the protein than the CRD is used for nucleic acid interactions. These observations support prior research suggesting that galectin3 is associated with riboneucleoprotein complexes and can act as a pre-mRNA splicing factor. Together, these studies show that galectin-3 can not only recognize carbohydrate moieties, but they suggest that through other epitopes it can act on the nuclear matrix to influence complex functions such as cell proliferation, morphology, and angiogenesis. These effects may be mediated by changes in the tissue matrix, nuclear matrix, and ultimately gene expression, or possibly directly by galectin-3.

\section{CONCLUSION}

We are just beginning to understand the functions of carbohydrate-protein interactions. Carbohydrates have the potential to store a wide range of biological information. Cellular recognition of this glycocode has been conserved 
TABLE I

Potential organizational roles of lectins
Known correlates in function

$\begin{array}{ll}\begin{array}{l}\text { Spatial location } \\ \text { Cellular health status } \\ \text { Rules of contact inhibition }\end{array} & \begin{array}{l}\text { L-selectin mediated binding of neutrophils to the endothelium [Honn and Tang, 1992] } \\ \text { Upregulation of L-selectin in inflammation [Munro et al., 1996] }\end{array} \\ \begin{array}{l}\text { Alterations in cell membrane glycoproteins modifies cell density and proliferation. [Wieser } \\ \text { and Oesch, 1988] } \\ \text { Stimulation and growth }\end{array} & \begin{array}{l}\text { Growth effects mediated by galectin-3 [Inohara et al., 1998] } \\ \text { Organized cell death }\end{array} \\ \text { Overexpression of galectin-3 blocks apoptosis [Akahani et al., 1997; Matarrese et al., 2000] }\end{array}$

over evolution and several mechanisms of carbohydrate binding have converged into having similar functions despite coming from disparate biologic origins. While smaller organisms may have used these lectins for identifying food sources in their environment, it has been suggested that more complex, multi-organ animals may use lectins for higher level organization [Kasai and Hirabayashi, 1996]. While many of the lectins have rather specific functions, the galectins have a broad range of complex functions making them interesting research targets. Studies utilizing molecular and cellular biology techniques have identified their specific involvement in cell proliferation, differentiation, and morphology. Due to our limited knowledge of the glycocode it is possible that our current techniques may be myopic and not able to fully describe the potential organizational functions of lectins in general (see Table I).

Spatial location has been alluded to in the model of neutrophil adhesion for extravasation into target tissues. It is well known that $\mathrm{L}$ selectin allows for the initial weak binding of neutrophils to the endothelium forming the cellular phenomenon known as "rolling." This is followed by the formation of a stronger bond formed by integrins. This sequence has also been described as "lock \& dock" by Honn and Tang [1992] and others. This model has often been referred to in understanding cancer cell metastasis, which appears to require a similar set of events for cancer cell extravasation. While this model describes a sequence for neutrophil binding, it is not clear whether this is an accurate model for the specificity of cancer cell adhesion and it leaves the question of how cells pass through the endothelium to target tissues. We would like to propose a model for "lock, dock, and entry" (Fig. 2).

The endothelial cells, upon which neutrophils and cancer cells initially bind, form a tight barrier along the blood vessels. Carbohydrate groups and L-selectin on the endothelial surface have been shown to play important roles in the initial steps of neutrophil adhesion. Studies have shown that L-selectin can be upregulated in both target tissues and in neutrophils during inflammatory responses [Munro et al., 1996].

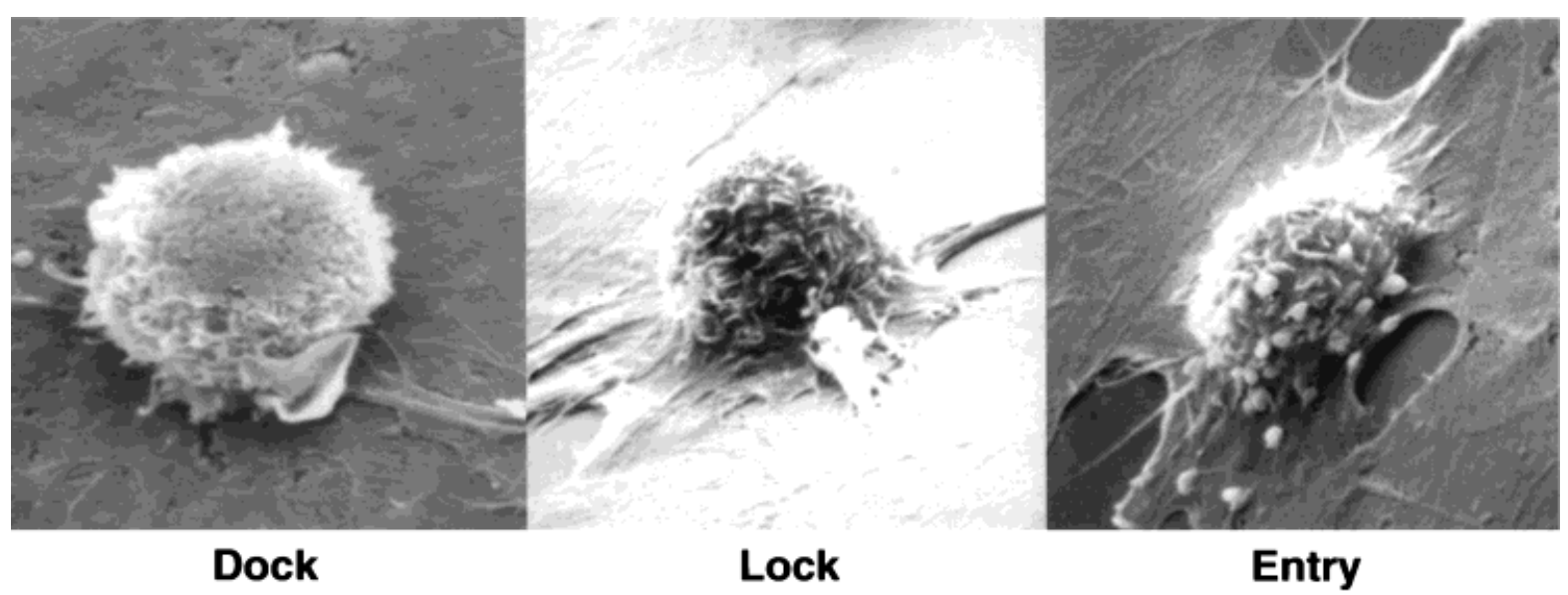

Fig. 2. "Dock, Lock, and Entry." This confocal microscopic image depicts the adhesion of a PC-3 cell to a monolayer of human bone marrow endothelial (HBME) cells. "Dock" refers to the initial lectin binding followed by "Lock", the more tight integrin binding. "Entry" refers to changes in the HBME cells retracting from the PC-3 cell, which is mediated through an unknown mechanism. 
Without a regulatory mechanism to increase affinity during an inciting event, binding would be non-specific or constitutive. In terms of the model for cancer cell metastasis, the problem is somewhat different. The target tissue may be perfectly healthy, but the cancer cell finds a way, without an inciting event, to bind specifically. One potential model would be if cancer cells could stimulate the regional production of L-selectin. This would take advantage of known mechanisms of neutrophil adhesion, but unlike neutrophil adhesion, it still leaves open the question of tissue specificity. Neutrophils potentially need to enter every tissue in the body whereas tumor cells often metastasize to specific tissues. The use of an L-selectinmediated pathway would implicate the potential for diffuse cancer cell adhesion. Another potential model would be if every different area of the vasculature bed had its own molecular address. This could be accomplished by carbohydrate presentation on the endothelial surface based on the ECM created by the underlying tissue. While initial adhesion may be nonspecific, it could be followed by a more specific event, such as "entry."

The model of "lock \& dock" only takes into account cellular adhesion to the endothelial cell. "Entry" requires passage between the endothelial cells. A model of "entry" involving destruction may presume that in response to cellular attachment, adhesion molecules may signal a change in the tissue matrix and allow for the release of enzymes such as metalloproteinases to degrade the ECM. While this may be plausible for cancer cells, this seems unlikely for neutrophils. This form of "entry." could produce inflammation and again cause an upregulation of L-selectin. An alternative model for "entry" involving communication would suggest that the response to adhesion may not be degradation, but instead signaling to the endothelial cells a response. This response could be mediated by protein-protein or carbohydrate-protein interactions. With the known effects of lectins on cellular morphology, it is plausible that a carbohydrate signal may cause an alteration in the tissue matrix of the endothelial cell to allow "entry." This signal could reflect molecular mimicry in presenting like carbohydrates, or the signal could indicate a command for "entry." While these events could be mediated by known protein-protein and protein-carbohydrate interactions there is no evidence that any known system alters endothelial cells to allow for "entry".

While extravasation by neutrophils and cancer cells represents a complex system, it is illustrative of the biologic use of carbohydrateprotein interactions and their potential breadth of involvement in regulating cellular function. Despite techniques gained through our understanding of DNA and proteins, understanding the glycocode and how to study it will be the next frontier. There are several obstacles that remain including understanding the mechanism of deciphering the signal and how cellular responses may then alter the presentation of carbohydrates. Linear codes can be translated in a linear fashion, but does the cell translate the three dimensional structure of carbohydrates in one or multi-dimensions? In addition, cellular responses may cause alterations in the presentation of subsequent carbohydrate moieties, but how long would this take and are there other targets besides post-transcriptional modifications in the Golgi complex? The answers to these questions will likely hold the key to cellular interactions on a molecular level as well as cellular organization. Not only will this have implications for the field of metastasis, but also organogenesis, tissue engraftment, and the study of responses to infection.

\section{REFERENCES}

Akahani S, Nangia-Makker P, Inohara H, Kim HR, Raz A. 1997. Galectin-3: a novel antiapoptotic molecule with a functional BH1 (NWGR) domain of Bcl-2 family. Cancer Res 57:5272-5276.

Arena N, Bodo M, Baroni T, Alia FA, Gaspa L, Becchetti E. 1990. Effects of lectins on cytoskeleton and morphology or cultured chick embryo fibroblasts. Cell Mol Biol 36: $317-328$.

Barondes SH. 1984. Soluble lectins: a new class of extracellular proteins. Science 223:1259-1264.

Berezney R, Coffey DS. 1974. Identification of a nuclear protein matrix. Biochem Biophys Res Comm 60:1410-1417.

Bissell MJ, Matie C, Laszlo A, Parry G. 1981. The influence of the extracellular matrix on normal and transformed phenotypes in culture. J Cell Biol 1:163a.

Brenner B, Gulbins E, Schlottmann K, Koppenhoefer U, Busch GL, Walzog B, Steinhausen M, Coggeshall KM, Linderkamp O, Lang F. 1996. L-selectin activates the Ras pathway via the tyrosine kinase p56lck. Proc Natl Acad Sci USA93:15376-15381.

Ciejek EM, Nordstrom JL, Tsai MJ, O’Malley BW. 1982. Ribonucleic acid precursors are associated with the chick oviduct nuclear matrix. Biochem 21:4945-4953.

Cooper DN, Barondes SH. 1990. Evidence for export of a muscle lectin from cytosol to extracellular matrix and for a novel secretory mechanism. J Cell Biol 110:1681-1691. 
Drickamer K. 1993. Evolution of $\mathrm{Ca}^{(2+)}$-dependent animal lectins. Progress in Nucleic Acid Res 232.

Drickamer K. 1995. Increasing diversity of animal lectin structures. Curr Opin Struct Biol 5:612-616.

Fey EG, Wan KM, Penman S. 1984. Epithelial cytoskeletal framework and nuclear matrix-intermediate filament scaffold: three-dimensional organization and protein composition. J Cell Biol 98:1973-1984.

Fulgenzi G, Graciotti L, Granata AL, Corsi A, Fucini P, Noegel AA, Kent HM, Stewart M. 1998. Location of the binding site of the mannose-specific lectin comitin on Factin. J Mol Biol 284:1255-1263.

Gabius HJ. 1994. Non-carbohydrate binding partners/ domains of animal lectins. Int J Biochem 26:469-477.

Gabius HJ. 1997. Animal lectins. Eur J Biochem 243:543576.

Gahmberg CG, Tolvanen M, Kotovuori P. 1997. Leukocyte adhesion-structure and function of human leukocyte beta2-integrins and their cellular ligands. Eur J Biochem 245:215-232.

Getzenberg RH, Pienta KJ, Huang EY, Murphy BC, Coffey DS. 1991. Modifications of the intermediate filament and nuclear matrix networks by the extracellular matrix. Biochem Biophys Res Comm 179:340-344.

Gospodarowicz D, Greenburg G, Birdwell CR. 1978. Determination of cellular shape by the extracellular matrix and its correlation with the control of cellular growth. Cancer Res 38:4155-4171.

Honn KV, Tang DG. 1992. Adhesion molecules and tumor cell interaction with endothelium and subendothelial matrix. Cancer Metastasis Rev 11:353-375.

Huber O, Korn R, McLaughlin J, Ohsugi M, Herrmann BG, Kemler R. 1996. Nuclear localization of beta-catenin by interaction with transcription factor LEF-1. Mech Dev 59:3-10.

Inohara H, Akahani S, Raz A. 1998. Galectin-3 stimulates cell proliferation. Exp Cell Res 245:294-302.

Isaacs JT, Barrack ER, Isaacs WB, Coffey DS. 1981. The relationship of cellular structure and function: the matrix system. Prog Clin Biol Res 75A:1-24.

Kaltner H, Stierstorfer B. 1998. Animal lectins as cell adhesion molecules. Acta Anat 161:162-179.

Kasai K, Hirabayashi J. 1996. Galectins: a family of animal lectins that decipher glycocodes. J Biochem 119:1-8.

Kornfeld S. 1992. Structure and function of the mannose 6phosphate/insulinlike growth factor II receptors. Annu Rev Biochem 61:307-330.

Lelievre SA, Bissell MJ. 1998. Communication between the cell membrane and the nucleus: role of protein compartmentalization. J Cell Biochem Suppl 30-31:250-263.

Lelievre SA, Weaver VM, Nickerson JA, Larabell CA, Bhaumik A, Petersen OW, Bissell MJ. 1998. Tissue phenotype depends on reciprocal interactions between the extracellular matrix and the structural organization of the nucleus. Proc Natl Acad Sci USA95:1471114716.

Matarrese P, Fusco O, Tinari N, Natoli C, Liu FT, Semeraro ML, Malorni W, Iacobelli S. 2000. Galectin-3 overexpression protects from apoptosis by improving cell adhesion properties. Int J Cancer 85:545-554.

Munro JM, Briscoe DM, Tedder TF. 1996. Differential regulation of leucocyte L-selectin (CD62L) expression in normal lymphoid and inflamed extralymphoid tissues. J Clin Pathol 49:721-727.

Nangia-Makker P, Honjo Y, Sarvis R, Akahani S, Hogan V, Pienta KJ, Raz A. 2000. Galectin-3 induces endothelial cell morphogenesis and angiogenesis. Am J Pathol 156:899-909.

Pienta KJ, Getzenberg RH, Coffey DS. 1991. Cell structure and DNA organization. Crit Rev Eu Gene Exp 1:355385 .

Pienta KJ, Murphy BC, Getzenberg RH, Coffey DS. 1993. The tissue matrix and the regulation of gene expression in cancer cells.JAI Press.

Ruoslahti E, Obrink B. 1996. Common principles in cell adhesion. Exp Cell Res 227:1-11.

Sato S, Hughes RC. 1992. Binding specificity of a baby hamster kidney lectin for $\mathrm{H}$ type I and II chains, polylactosamine glycans, and appropriately glycosylated forms of laminin and fibronectin. J Biol Chem 267:69836990.

Sato S, Hughes RC. 1994. Regulation of secretion and surface expression of Mac-2, a galactoside-binding protein of macrophages. J Biol Chem 269:4424-4430.

Sharon N, Lis H. 1997. Glycoproteins: structure and function. London; New York: Chapman \& Hall.

Traub P. 1985. Are intermediate filament proteins involved in gene expression. Ann N Y Acad Sci 455:68-78.

Villalobo A, Gabius H. 1998. Signaling pathways for transduction of the initial message of the glycocode into cellular responses. Acta Anat 161:110-129.

Vogelstein B, Pardoll DM, Coffey DS. 1980. Supercoiled loops and eucaryotic DNA replicaton. Cell 22:79-85.

Wang L, Inohara H, Pienta KJ, Raz A. 1995. Galectin-3 is a nuclear matrix protein which binds RNA. Biochem Biophys Res Comm 217:292-303.

Weiser RJ, Oesch F. 1988. Contact-dependent regulation of growth of diploid human fibroblasts is dependent upon the presence of terminal galactose residues on plasma membrane glycoproteins. Exp Cell res 176: 80-86.

Zanetta JP. 1997. Lectins and carbohydrates in animal cell adhesion and control of cell proliferation. London; New York: Chapman \& Hall. 